\title{
Occult pulmonary arterial hypertension in patients with previous pulmonary tuberculosis
}

\author{
I S Kalla, MB BCh, PhD, FCP, FCCP (USA), Cert Pulm (SA), Cert Critical Care (SA); A Miri, BSc, MB ChB; \\ F Seedat, MB ChB, FCP, MMed (Int Med) \\ Division of Pulmonology, Department of Internal Medicine, School of Clinical Medicine, Faculty of Health Sciences, University of the Witwatersrand, \\ Johannesburg, South Africa
}

Corresponding author: I S Kalla (laeeka@telkomsa.net; iskalla786@gmail.com)

\begin{abstract}
Background. Pulmonary tuberculosis (TB) still causes a significant public healthcare burden. Despite successful treatment, TB can lead to permanent lung damage and pulmonary hypertension ( $\mathrm{PH})$. $\mathrm{PH}$ can also occur in the absence of significant lung damage, leading clinicians to question whether pulmonary TB may cause pulmonary arterial hypertension (PAH), an entity that has not been otherwise described.

Objectives. To determine the prevalence of PAH in patients previously treated for TB.

Methods. We recruited 20 participants who were previously treated for TB and had no other underlying risk factors for the development of PH. The participants underwent electrocardiography (ECG), chest radiography, lung function tests and echocardiography (ECHO). Data from these non-invasive investigations were evaluated to determine findings that were suggestive of $\mathrm{PH}$.

Results. At a median duration of 30 months from diagnosis of TB, no participant had echocardiography findings that were suggestive of $\mathrm{PH}$ (pulmonary artery pressure (PAP) $\geq 40 \mathrm{mmHg}$ ). However, there was a negative correlation between the time from diagnosis and right ventricular dysfunction assessed by measuring a tricuspid annular plane systolic excursion $(r=-0.5136 ; p=0.0205)$. Furthermore, one-third of the participants $(n=7)$ had one or more ECG features supporting PH and $85 \%$ of the participants $(n=17)$ demonstrated at least one chest $\mathrm{X}$-ray (CXR) feature of $\mathrm{PH}$.

Conclusion. Although our study did not demonstrate ECHO findings supporting PH, ECG and CXR modalities were suggestive. Therefore, future studies consisting of larger cohorts and including the use of other sensitive modalities such as computed tomography are warranted. Moreover, these studies will need to determine whether the entity of PAH secondary to previously treated pulmonary TB exists.
\end{abstract}

Afr J Thoracic Crit Care Med 2020;26(4):133-137. https://doi.org/10.7196/AJTCCM.2020.v26i4.110

Tuberculosis (TB) is ranked amongst the top 10 leading causes of death worldwide and is the leading cause of death from a single infectious agent. ${ }^{[1]}$ South Africa (SA) has a high burden of TB, with $\sim 438000$ new cases reported in 2019. ${ }^{[2]}$ The Department of Health in SA has implemented strategies that have been successful in curbing the incidence of TB. ${ }^{[3]}$

Despite successful treatment, TB may cause significant long-term cardiorespiratory complications that are well known, including fibrocavitary changes, bronchiectasis, chronic pulmonary aspergillosis and chronic obstructive pulmonary disease. ${ }^{[4]}$ These complications may have further sequelae such as the development of pulmonary hypertension (PH) and right heart failure, which substantially impacts quality of life and further burdens the healthcare system. ${ }^{[5]}$

Pulmonary hypertension is defined as an increase in mean pulmonary artery pressure (PAP) $\geq 20 \mathrm{mmHg}$ at rest as assessed by right heart catheterisation (RHC) as gold standard. ${ }^{[6,7]}$ Other modalities such as echocardiography may be used to determine the probability of $\mathrm{PH} .{ }^{[6]}$ This disease is associated with significant morbidity and mortality ${ }^{[8]}$ Therefore, it is crucial that those suspected to be at risk of disease are identified and be put on treatment as early as possible.

An under-recognised cause of $\mathrm{PH}$ associated with previously treated TB is the development of a vasculitis of the pulmonary artery. ${ }^{[9]}$ This may lead to an increase in pulmonary arterial vascular resistance and subsequently $\mathrm{PH} .{ }^{[10]}$ Other mechanisms that have been described to lead to the development of $\mathrm{PH}$ include obliterative changes of the pulmonary arteries and an endarteritis obliterans in the vessels following $\mathrm{TB},{ }^{[11]}$ similar to those noted in the development of pulmonary arterial hypertension (PAH).

Specific targeted therapy has recently been developed for PAH based on its underlying pathophysiology. These include endothelin receptor antagonists, phosphodiesterase type 5 inhibitors, prostacyclin analogues and prostacyclin receptor agonists. ${ }^{[6]}$ Early and appropriate administration of these drugs has been shown to be efficacious in alleviating symptoms, improving the haemodynamic profile and delaying the time to worsening of clinical symptoms. ${ }^{[12]}$ Many of these therapies are not currently available in the SA public healthcare sector due to their costs.

Previous studies have demonstrated that PAH can develop in patients with minimal lung complications in countries with a high burden of TB. ${ }^{[13,14]}$ In fact, Allwood et al ${ }^{[14]}$ noted that significant destruction of the pulmonary vasculature, in the absence of extensive parenchymal disease following $\mathrm{TB}$, is an unexplored entity and may result in post-TB associated PAH. ${ }^{[14]}$ They suggested that this paucity in the literature underscores a need for future studies to resolve this challenge. The outcomes of these studies will have serious implications on the burden of TB disease, the need for early detection of PAH and 
the need to dispense appropriate treatment in order to minimise disease morbidity. The aim of this study was to investigate whether $\mathrm{PAH}$ was present in participants who were previously treated for TB.

\section{Methods}

Participants who were previously treated for TB were recruited and enrolled into this pilot prospective cohort study between October 2018 and April 2019. The study was undertaken in the Department of Infectious Diseases at Charlotte Maxeke Johannesburg Academic Hospital (CMJAH), a quaternary hospital in Gauteng Province, SA. Participants were identified from the TB registry kept in the Outpatient Department or from those who attended follow-up visits after completing their TB treatment.

Participants with active TB and those with known aetiologies of $\mathrm{PH}$ such as HIV, chronic obstructive pulmonary disease, interstitial lung disease, obstructive sleep apnoea, autoimmune diseases, connective tissue disorders, collagen vascular disorders, liver cirrhosis, chronic bilharzia, heart disease and current or previous pulmonary embolus or deep vein thrombosis were excluded from the study. A chest X-ray (CXR) was subsequently performed to assess the presence of parenchymal lung disease. Participants who had severe bronchiectasis were risk stratified using the FACED score developed by Martinez-Garcia et al. ${ }^{[15]}$ The score uses the forced expiratory volume in one second $\left(\mathrm{FEV}_{1}\right)$, age, chronic microbial colonisation, radiological extent and dyspnoea to stratify patients with non-cystic bronchiectasis. Patients with a FACED score $\geq 5$ were excluded from the study.

A structured questionnaire was used to record demographics, details of TB diagnosis (date, method and duration of therapy) and TB drug sensitivity. Each participant underwent an electrocardiogram (ECG), pulmonary function test (spirometry and diffusing capacity of lung for carbon monoxide (DLCO)), echocardiography (ECHO) and CXR to determine features that were indicative of PAH. The data were interpreted by a pulmonologist in the Division of Pulmonology at CMJAH. A diagnosis of $\mathrm{PH}$ was made based on a peak systolic pulmonary artery pressure (PASP) $\geq 40 \mathrm{mmHg}$ on ECHO. ${ }^{(6)}$ The gold standard for the diagnosis of PAH is RHC. However,
RHC is a highly invasive procedure that is infrequently performed in our setting due to resource constraints. Therefore, to ensure the safety of participants and to preserve resources, RHC was not performed.

This study was approved by the Human Research Ethics Committee of the University of the Witwatersrand (ref. no. M180275). All participants provided written informed consent.

Frequencies, median and interquartile range were used to describe demographic characteristics, clinical features and investigations (Tables 1 and 2). We explored pairwise associations between numeric variables to determine correlation with each other and with the outcomes of the tricuspid annular plane systolic excursion (TAPSE) and PASP. We plotted linear fit prediction plots with confidence intervals to further explore statistically significant pairwise associations of importance (Fig. 1). Statistical analysis was performed using the SPSS software, version 12 (SPSS Inc., USA).

\section{Results}

We recruited and enrolled 22 participants who were previously treated for smearpositive TB into the study. However, we excluded two participants who had severe bronchiectasis. The median age of the remaining 20 participants was 33 years old

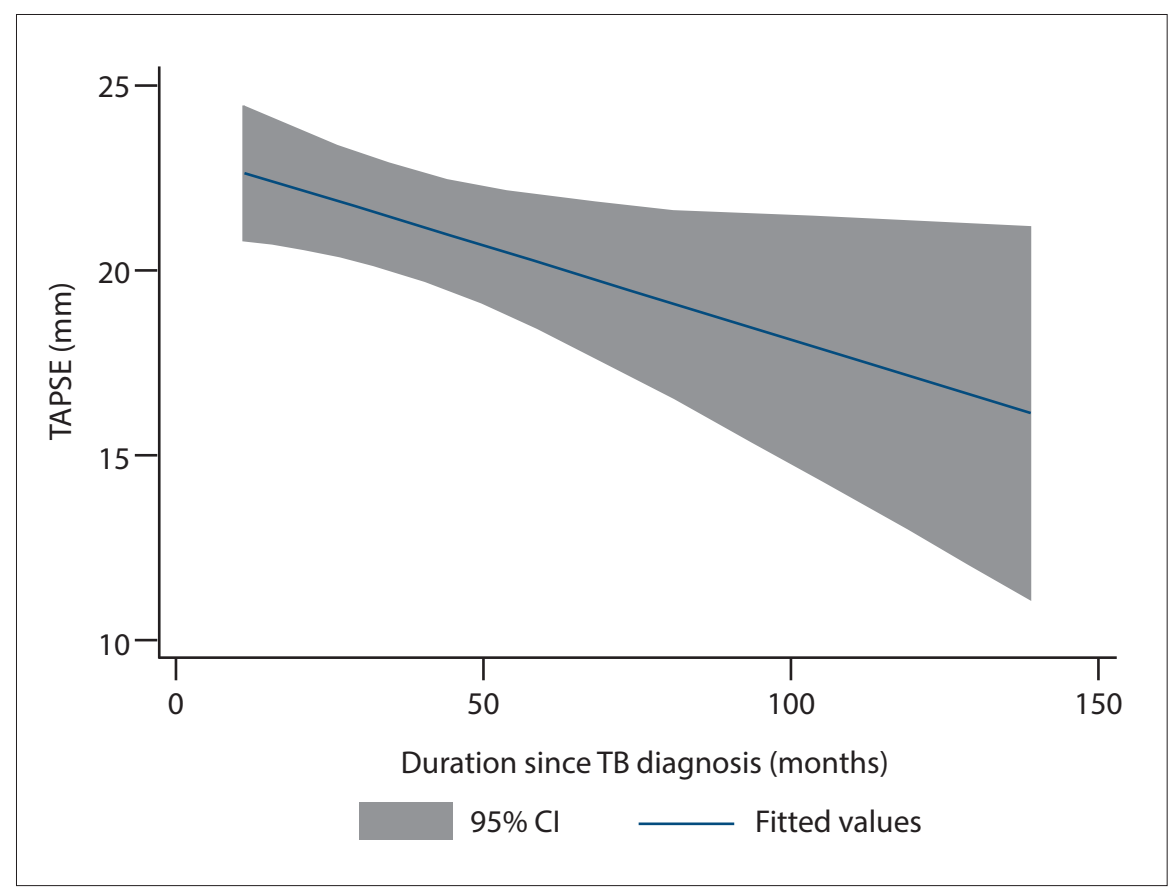

Fig. 1. Linear prediction plot showing association between duration since TB diagnosis and tricuspid annular plane systolic excursion (TAPSE). (CI= confidence interval; $T B=$ tuberculosis. $)$ (interquartile range (IQR) 29 - 41.5). The majority of the participants $(60 \%)$ were female and black African (Table 1). All participants had microbiologically confirmed TB at diagnosis, with $80 \%(n=16)$ by sputum, $15 \%(n=3)$ by bronchial washings and $5 \%(n=1)$ by computed tomography (CT) guided fine needle aspirate (FNA) of a lung nodule. The median duration after initial TB diagnosis was 30 months (IQR 14 - 42). No participants had a past or current history of smoking tobacco.

A few participants $(15 \% ; n=3)$ complained of a dry persistent non-productive cough and no complaints of dyspnoea, sputum expectoration or haemoptysis were reported.

Pulmonary function tests showed a median forced expiratory volume in one second: forced vital capacity $\left(\mathrm{FEV}_{1}: \mathrm{FVC}\right)$ ratio of $83 \%$ (IQR $73-87$ ). We also found that in $15 \%(n=3)$ of the participants, the DLCO was reduced after an average of 23 months following diagnosis of TB. All the participants had room air saturation measurements that were above $95 \%$ by pulse oximetry (Table 2 ). More than one-third of the participants (35\%; $n=7)$ had one or more features of PH on ECG while the majority of the participants $(85 \%$; $n=17$ ) demonstrated at least one CXR feature suggestive of $\mathrm{PH}$ (Table 2). The majority of the patients (60\%) had some degree of bronchovascular distortion and pleuro-parenchymal 
Table 1. Characteristics of patients with previous TB $(N=20)$

\section{Patient characteristics}

Demographic characteristics

Overall age in years

Mean (SD) ${ }^{\star} \quad n(\%)$

Median (IQR)

$36.65(12.83)$

$33(29-41.5)$

Self-reported race

$\begin{array}{ll}\text { Asian } & 1(5) \\ \text { Black } & 12(60) \\ \text { Coloured } & 1(5) \\ \text { Indian } & 5(25) \\ \text { White } & 1(5) \\ \text { Gender } & \\ \text { Male } & 8(40) \\ \text { Female } & 12(60)\end{array}$

Clinical features

Details regarding prior TB infection

Duration since TB diagnosis (months) 35.65 (31.02)

Median (IQR)

$30(14-42)$

Mode of TB diagnosis

Sputum

Bronchial washings

CT-guided FNA lung nodule

(CXR suggestive of active TB)

Current presence of respiratory

symptoms

Cough

Dyspnoea

Sputum expectoration

0

0

Haemoptysis

Comorbidities and risk factors

Smoking

0

Hypertension

Diabetes

Malignancy

Screening for sleep-disordered breathing

Neck circumference $>40 \mathrm{~cm}$

Overweight (BMI 25 - 30)

Obesity (BMI $>30$ )

Snoring

Age $>55$

Male sex

$8(40)$

$\mathrm{SD}=$ standard deviation; $\mathrm{IQR}=$ interquartile range; $\mathrm{FNA}=$ fine-needle aspiration; $\mathrm{CXR}=$ chest $\mathrm{X}$-ray; $\mathrm{BMI}=$ body mass index

*Unless otherwise specified.

bands on CXR and the rest had no radiological complications of TB (Table 2). We found no participants that displayed ECHO criteria supportive of PH based on PASP at a median of 30 months after TB diagnosis (Table 3). However, the overall trend of TAPSE values was observed to decline over time after the initial TB diagnosis (Table 2).

\section{Discussion}

In this pilot study, we examined PAH in HIV-negative participants that were previously diagnosed with TB. We found CXR and ECG changes that were suggestive of $\mathrm{PAH}$ in some participants after a
Table 2. Clinical investigations

\begin{tabular}{|c|c|c|}
\hline Findings on ECG & Median (IQR) & $n(\%)$ \\
\hline $\mathrm{P}$ & & 0 \\
\hline Right-axis deviation & & $1(5)$ \\
\hline S wave in standard lead 1 & & $3(15)$ \\
\hline Q wave in standard lead 3 & & $2(10)$ \\
\hline T wave in standard lead 3 & & $1(5)$ \\
\hline $\mathrm{R}$ wave in ventricular lead 1 & & $1(5)$ \\
\hline $\mathrm{RVH}$ & & 0 \\
\hline
\end{tabular}

RV strain

RBBB

0

Findings on chest X-ray

Elevated cardiac apex

$10(50)$

Enlarged right atrium

Enlarged pulmonary arteries

Pruning of peripheral pulmo

nary vessels

Pleuro-parenchymal bands

Volume loss

Tracheal deviation

Spirometry

FEV

FVC

$2.77(2.29-3.31)$

$3.41(2.82-3.96)$

Ratio

DLCO (\%Pred)

$82.85(73.10-86.85)$

99.5 (84.5 - 108.5)

Low DLCO

Room air saturation

$96(95.5$ - 97.0)

Echocardiography

LVIDd (mm)

LVIDs (mm)

LVEF (\%)

RWMA

0

Left atrium $(\mathrm{mm})$

$43.5(41.5-48.5)$

$28(27.5-30)$

$60(56-64)$

$27.5(23-31)$

$23(21-26.5)$

$1.24(1-1.50)$

$6(5.19-8.30)$

Aortic regurgitation

Mitral regurgitation

Tricuspid regurgitation

TAPSE (mm)

$21(19-23)$

TAPSE $<16 \mathrm{~mm}$

0

PASP $(\mathrm{mmHg})$

$18(8.5$ - 24.5)

RAP (mmHg)

$5(3-9.5)$

IVC (mm)

$14(13-18)$

NT-proBNP

$26(16-66)$

ECG = electrocardiography; $\mathrm{IQR}$ = interquartile range; $\mathrm{P}=$ pulmonale; $\mathrm{R}=$ right-axis deviation $\mathrm{RVH}=$ right ventricular hypertrophy; $\mathrm{RV}=$ right ventricular; $\mathrm{RBBB}=$ right bundle branch block; $\mathrm{FEV}_{1}=$ forced expiratory volume in one second; $\mathrm{FVC}=$ forced vital capacity; $\mathrm{DLCO}=$ diffusing capacity of lung for carbon monoxide; LVIDd = left ventricular internal diameter end diastole; LVIDs = left ventricular internal diameter end systole; LVEF = left ventricular ejection fraction; RWMA = regional wall motion abnormality; TAPSE = tricuspid annular plane systolic excursion; PASP = pulmonary hypertension echocardiography; RAP = right arterial pressure; IVC = inferior vena cava; $\mathrm{NT}$-proBNP $=\mathrm{N}$-terminal pro-B-type natriuretic peptide. 
Table 3. Radiological features of the lung scarring chest X-ray $n(\%)$

Right lung

Right upper lobe/zone

$10(50)$

Right mid-zone

$1(5)$

Right lower lobe/zone

$4(20)$

Left lung

Left upper lobe/zone

Left mid-zone

$1(5)$

Left lower zone

$1(5)$

Diffuse fibro-cavitary changes

$1(5)$

No fibro-cavitary changes

$8(40)$

Fibro-cavitary changes limited to one lobe

$6(30)$

Fibro-cavitary changes in two lobes

$3(15)$

Fibro-cavitary changes in three lobes

median duration of 30 months after TB diagnosis. However, no ECHO features of PAH were noted in all participants. A decline in TAPSE was observed over time after TB diagnosis, suggesting a possible decline in right ventricular function.

CXR is readily used as a screening tool for PAH, particularly in low-resource settings and is also able to assess lung parenchymal changes. ${ }^{[16]}$ CXR has a high sensitivity (96.9\%) and specificity (99.1\%) for detection of $\mathrm{PAH}$ when pre-test probability is $50 \%$ or more. ${ }^{[17]}$ Miniati et al. ${ }^{[17]}$ suggested that CXR findings that are suggestive of $\mathrm{PAH}$ are sufficient to warrant further investigation by RHC and that a normal CXR does not exclude the presence of PH. The severity of $\mathrm{PH}$, however, cannot be correlated with CXR changes. ${ }^{[16]}$ We showed in this study that $85 \%$ of the participants had CXR features that were suggestive of $\mathrm{PH}$. Although the pre-test probability in these patients was low, these findings still indicated a possible need for further investigations, which would include more definitive investigations such as chest computed tomography (CT) scan and RHC for the confirmation of PAH. This would offer this subgroup of patients novel therapies for PAH under a trial scenario to observe outcomes.

ECG is also another cost-effective screening tool that is not sufficiently sensitive or specific for the diagnosis of $\mathrm{PH}$. For instance, a previous study showed that ECG can be used to detect right ventricular hypertrophy and right-axis deviation in patients with PAH $87 \%$ and $79 \%$ of the time, respectively. ${ }^{[18]}$ Another study conducted by Al-Naamani et al. ${ }^{[17]}$ demonstrated a positive predictive value that was $>80 \%$ in specific ECG criteria, namely R/S amplitude in V1 $>1$ and right-axis deviation of QRS axis $>110^{0} .{ }^{[19]}$ The absence of ECG features does not exclude the presence of $\mathrm{PAH} .{ }^{[18]}$ Our study found right-axis deviation in one participant and a dominant $\mathrm{R}$ wave in $\mathrm{V} 1$.

Transthoracic ECHO is used as a screening tool for suspected $\mathrm{PH}$ in individuals with suggestive signs and symptoms. It determines the probability of $\mathrm{PH}$ and has a sensitivity of $83 \% ;{ }^{[20]}$ however, detection of mild PH is limited. ${ }^{[21]}$ PASP is estimated from the peak tricuspid regurgitant jet velocity and the right ventricular size and function is measured by TAPSE. ${ }^{[22]}$ The accuracy of transthoracic ECHO in estimating PASP has been questioned as it may frequently underestimate PASP. ${ }^{[23]}$ This has been attributed to inaccuracies in the estimation of right atrial pressure and poor Doppler imaging of the trans-tricuspid regurgitant jet. ${ }^{[23]}$

The measurement of the tricuspid regurgitant velocity (TRV) by echocardiography improves the probability of detecting PAH. An elevated TRV $(>3.4 \mathrm{~m} / \mathrm{s})$ suggests a high probability of PAH. We did not include TRV in the analysis for this study, and this is a potential limitation of the study.

The trend of a decreasing TAPSE over time observed in our study highlighted the potential development of PAH over time from initial TB diagnosis. Perhaps the lack of ECHO findings that were suggestive of $\mathrm{PAH}$ in this study was due to $\mathrm{PAH}$ developing later than the median time of 30 months (IQR 14 - 42). A study undertaken by Humbert et al. ${ }^{[24]}$ found a 27 -month delay between onset of symptoms and diagnosis of PAH, with 75\% of patients having New York Heart Association functional class III at time of diagnosis. ${ }^{[24]}$ This suggested that these individuals may need further prospective follow-up of RV function as well as the performance of more detailed ECHO measures such as TRV to determine if PAH is present.

CT is becoming more accepted as an initial test in the evaluation of $\mathrm{PH} .{ }^{[16,25]}$ A main pulmonary artery diameter (PAD) $\geq 29 \mathrm{~mm}$ has an $87 \%$ sensitivity in the diagnosis of $\mathrm{PH} .{ }^{[21]}$ The size of the pulmonary artery measured on CT is positively correlated with the severity of

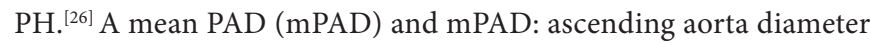
(AAD) ratio $>1$ has been shown to have a high correlation $(r=0.51$ and $r=0.53$, respectively; $p<0.001)$ with PAP. When an increased $\mathrm{mPAD}$ is accompanied by a segmental artery-to-bronchus ratio that is $>1: 1$ in 2 or 4 pulmonary lobes, sensitivity in the diagnosis of $\mathrm{PH}$ is $100 \% .{ }^{[26]} \mathrm{A}$ mPAD:AAD ratio $>1$ has a sensitivity of $70.8 \%$ and a specificity of $76.5 \%$ for the diagnosis of $\mathrm{PH}$. The positive predictive value of mPAD: AAD ratio $>1$ for the diagnosis of $\mathrm{PH}$ is $96 \% \cdot{ }^{[27]} \mathrm{CT}$ is further useful in identifying other causes of $\mathrm{PH}$ such as pulmonary vasculature, lung parenchyma and cardiovascular structures. ${ }^{[28]}$

One of the major limitations of this pilot study was the small number of participants that were recruited and enrolled in the study. Identifying participants who were previously treated for TB and had no underlying risk factors for the development of PAH is difficult in a quaternary hospital where most patients suffer from several co-existing pathologies. A more detailed ECHO evaluation of $\mathrm{PAH}$ combined with CT may be of value in future studies. Finally, the duration from time of TB diagnosis to enrolment may have been too short to identify the occurrence of PAH. Therefore, this period should be extended in future studies to ensure that sufficient time is provided for PAH to develop.

\section{Conclusion}

Although we did not determine ECHO findings that were suggestive of PAH in the HIV-negative participants that were previously treated for TB, we did find the presence of CXR and ECG features which were suggestive of $\mathrm{PH}$ and the possible presence of $\mathrm{PAH}$. This provides enough evidence to prompt further studies with larger sample sizes, a more heterogeneous post-TB population and inclusion of more indepth ECHO analysis to evaluate RV function in combination with radiological studies such as $\mathrm{CT}$ to examine the occurrence of $\mathrm{PAH}$ after TB.

Declaration. None.

Acknowledgements. None. 
Author contributions. AM conducted the study, interpreted the data and wrote the manuscript. ISK and FS conceptualised the study, assisted with data interpretation and revised the manuscript. All the authors approved the final manuscript for publication.

Funding. None.

Conflicts of interest. None.

1. Mello FCQ, Silva DR, Dalcolmo MP. Tuberculosis: Where are we? J Bra de Pneumol 2018;44(2):82. https://doi.org/10.1590/s1806-37562017000000450

2. Vos T, Lim SS, Abbafati C, et al. Global burden of 369 diseases and injuries in 204 countries and territories, 1990 - 2019: A systematic analysis for the global burden of disease study 2019. Lancet 2020;396(10258):1204-1222.

3. Glaziou P, Floyd K, Raviglione MC. Global epidemiology of tuberculosis. Semin Respir Crit Care Med 2018;39(3):271-285. https://doi.org/10.1101\%2Fcshperspect. a017798

4. Shah M, Reed C. Complications of tuberculosis. Curr Opin Infect Dis 2014;27(5):403410. https://doi.org/10.1097/QCO.0000000000000090

5. Akkara SA, Shah AD, Adalja M, Akkara AG, Rathi A, Shah DN. Pulmonary tuberculosis: The day after. Int J Tuberc Lung Dis 2013;17(6):810-813. https://doi. org/10.5588/ijtld.12.0317

6. Galie N, Humbert M, Vachiery JL, et al. 2015 ESC/ERS Guidelines for the diagnosis and treatment of pulmonary hypertension: The joint task force for the diagnosis and treatment of pulmonary hypertension of the European Society of Cardiology (ESC) and the European Respiratory Society (ERS): Endorsed by Association for European Paediatric and Congenital Cardiology (AEPC), International Society for Heart and Lung Transplantation (ISHLT). Eur Heart J 2016;37(1):67-119. https://doi. org/10.1093/eurheartj/ehv317.

7. Simonneau G, Montani D, Celermajer DS, et al. Haemodynamic definitions and updated clinical classification of pulmonary hypertension. Eur Respir J 2019;53(1):1801913. https://doi.org/10.1183/13993003.01913-2018

8. Chang WT, Weng SF, Hsu CH, et al. Prognostic factors in patients with pulmonary hypertension - a nationwide cohort study. J American Heart Assoc 2016;5(9). https:// doi:10.1161/JAHA.116.003579

9. Kim HY, Song KS, Goo JM, Lee JS, Lee KS, Lim TH. Thoracic sequelae and complications of tuberculosis. Radiographics 2001;21(4):839-858. https://doi. org/10.1148/radiographics.21.4.g01j106839.

10. Montani D, Günther S, Dorfmüller P, et al. Pulmonary arterial hypertension. Orphanet J Rare Dis 2013;8:97. https//doi.10.1186/1750-1172-8-97

11. Cudkowicz L. The blood supply of the lung in pulmonary tuberculosis. Thorax 1952;7(3):270-276. https://doi.org/10.1136\%2Fthx.7.3.270

12. Montani D, Chaumais MC, Guignabert C, et al. Targeted therapies in pulmonary arterial hypertension. Pharmacol Ther 2014;141(2):172-191.

13. Verma AK. Tuberculosis and pulmonary hypertension: Commentary. Lung India 2016;33(2):232-233. https://doi.org/10.1016/j.pharmthera.2013.10.002
14. Allwood BW, Maarman GJ, Kyriakakis CG, Doubell AF. Post-pulmonary tuberculosis complications in South Africa and a potential link with pulmonary hypertension: Premise for clinical and scientific investigations. S Afr Med J 2018;108(7):12339. https://doi.org/10.7196/samj.2018.v108i7.13359

15. Martínez-García MÁ, De Gracia J, Relat MV, et al. Multidimensional approach to noncystic fibrosis bronchiectasis: The FACED score. Eur Respir J 2014;43(5):1357-1367. https://doi:10.1183/09031936.00026313

16. Ascha M, Renapurkar RD, Tonelli AR. A review of imaging modalities in pulmonary hypertension. Annals Thorac Med 2017;12(2):61-73. https://doi.org/10.4103/18171737.203742

17. Miniati M, Monti S, Airo E, et al. Accuracy of chest radiography in predicting pulmonary hypertension: A case-control study. Thromb Res 2014;133(3):345-351. https://doi.org/10.1016/j.thromres.2013.12.019

18. Forfia PR, Trow TK. Diagnosis of pulmonary arterial hypertension. Clin Chest Med 2013;34(4):665-681. https//doi.10.1016/j.ccm.2013.09.001.

19. Al-Naamani K, Hijal T, Nguyen V, Andrew S, Nguyen T, Huynh T. Predictive values of the electrocardiogram in diagnosing pulmonary hypertension. Int J Cardiol 2008;127(2):214-218. https://doi.org/10.1016/j.ijcard.2007.06.005

20. Janda S, Shahidi N, Gin K, Swiston J. Diagnostic accuracy of echocardiography for pulmonary hypertension: A systematic review and meta-analysis. Heart 2011;97(8):612-622. https://doi.org/10.1136/hrt.2010.212084

21. Sirajuddin A, Donnelly EF, Crabtree TP, et al. ACR appropriateness criteria suspected pulmonary hypertension. J Am Coll Radiol 2017;14(5s):S350-S361. https://doi. org/10.1016/j.jacr.2017.01.040

22. Ahmed M, Dweik RA, Tonelli AR. What is the best approach to a high systolic pulmonary artery pressure on echocardiography? Cleveland Clin J Med 2016;83(4):256-260. https://doi.org/10.3949/ccjm.83a.14186

23. Fisher MR, Forfia PR, Chamera E, et al. Accuracy of Doppler echocardiography in the hemodynamic assessment of pulmonary hypertension. Am J Respir Crit Care Med 2009;179(7):615-621. https://doi.org/10.1164\%2Frccm.200811-1691OC

24. Humbert M, Sitbon O, Chaouat A, et al. Pulmonary arterial hypertension in France: Results from a national registry. Am J Resp Crit Care Med 2006;173(9):1023-1030. https://doi.org/10.1164/rccm.200510-1668oc

25. Mahammedi A, Oshmyansky A, Hassoun PM, Thiemann DR, Siegelman SS, Pulmonary artery measurements in pulmonary hypertension: The role of computed tomography. J Thorac Imaging 2013;28(2):96-103. https://doi.org/10.1097/ rti.0b013e318271c2eb

26. Tan RT, Kuzo R, Goodman LR, Siegel R, Haasler GB, Presberg KW. Utility of CT scan evaluation for predicting pulmonary hypertension in patients with parenchymal lung disease. Medical College of Wisconsin Lung Transplant Group. Chest 1998;113(5):1250-1256. https://doi.org/10.1378/chest.113.5.1250

27. Ng CS, Wells AU, Padley SP. A CT sign of chronic pulmonary arterial hypertension: The ratio of main pulmonary artery to aortic diameter. J Thorac Imaging 1999;14(4):270278. https://doi.org/10.1097/00005382-199910000-00007

28. Altschul E, Remy-Jardin M, Machnicki S, et al. Imaging of pulmonary hypertension: Pictorial essay. Chest 2019;156(2):211-227. https://doi:10.1016/j.chest.2019.04.003

Accepted 9 October 2020. 